\title{
Automated road marking detection system for autonomous car
}

\begin{abstract}
In recent years, road markings detection has received great attention and has been widely explored due to the aim of producing a system that is able to detect various shape of road markings on the images that are captured under various imaging conditions. Generally, the road images are captured using a camera, which has been placed inside the vehicle at a fixed position. However, the quality of the resulting images decreases if the camera position has been changed accidentally, due to the movement of the car. Hence, in this paper, a road markings detection system that tackle the problems of detecting road markings on the images captured under various camera positions and illumination conditions is proposed. The system consists of a graph cut segmentation method, which is used to detect the road, an inverse perspective transform method, which is used to convert the image into a bird's-eye view image, an image normalization method, which is CLAHE and a connected component analysis that is used to remove the background. We demonstrate the usefulness of the constructed algorithm by performing experiments on a database that consists of 400 road images.
\end{abstract}

Keyword: Binary image; Bird's eye view image; CLAHE; Road detection 